\title{
LINEAR SUPERPOSITIONS WITH MAPPINGS WHICH LOWER DIMENSION
}

BY

\section{Y. STERNFELD}

\begin{abstract}
It is shown that for every $n$-dimensional compact metric space $X$, there exist $2 n+1$ functions $\left\{\varphi_{j}\right\}_{j=1}^{2 n+1}$ in $C(X)$ and $n$ mappings $\left\{\psi_{i}\right\}_{i=1}^{n}$ on $X$ with 1-dimensional range each, with the following property: for every $0 \leqslant k \leqslant n$, every $k$ tuple $\left\{\psi_{i_{l}}\right\}_{l=1}^{k}$ of the $\psi_{i}$ 's, and every $2(n-k)+1$ tuple $\left\{\varphi_{j_{m}}\right\}_{m=1}^{2(n-k)+1}$ of the $\varphi_{j}$ 's, each $f \in C(X)$ can be represented as $f(x)=\sum_{l=1}^{k} g_{l}\left(\psi_{i}(x)\right)+\sum_{m=1}^{2(n-k)+1} h_{m}\left(\varphi_{j_{m}}(x)\right)$, with $g_{l} \in C\left(\psi_{i}(X)\right)$ and $h_{m} \in C(R)$.

It is also shown that in many cases the number $2(n-k)+1$ is the smallest possible.
\end{abstract}

1. Introduction. Let $X$ and $Y_{i}, 1 \leqslant i \leqslant k$, be compact metric spaces, and let $\varphi_{i}$ : $X \rightarrow Y_{i}$ be continuous functions. The family $\left\{\varphi_{i}\right\}_{1 \leqslant i \leqslant k}$ is said to be a measure separating family if each $f \in C(X)$ admits a representation

$$
f(x)=\sum_{i=1}^{k} g_{i}\left(\varphi_{i}(x)\right)
$$

with $g_{i} \in C\left(Y_{i}\right), 1 \leqslant i \leqslant k$.

$\left\{\varphi_{i}\right\}_{1 \leqslant i \leqslant k}$ is said to be a uniformly (point) separating family if each $f \in l_{\infty}(X)$ admits a representation (*) with $g_{i} \in l_{\infty}\left(Y_{i}\right), 1 \leqslant i \leqslant k$. $\left(C(X)\left(\right.\right.$ resp. $\left.l_{\infty}(X)\right)$ is the Banach space of real continuous (resp. bounded) functions on $X$ with the sup norm.)

As shown in [9] $\left\{\varphi_{i}\right\}_{1 \leqslant i \leqslant k}$ is measure separating (resp. uniformly separating) if and only if there exists a positive $\lambda$, such that for each $\mu \in M(X)$ (resp. $\mu \in l_{1}(X)$ ) there correspond some $1 \leqslant i \leqslant k$ so that

$$
\left\|\mu \circ \varphi_{i}\right\| \geqslant \lambda\|\mu\| \text {. }
$$

(Here $M(X)$ is the Banach space of real Borel measures on $X$ with the total variation as the norm; $l_{1}(X)$ is the subspace of $M(X)$ which consists of the purely atomic measures. Given an element $\mu$ of $M(X)$ (resp. $\left.l_{1}(X)\right) \mu \circ \varphi_{i}$ is the element of $M\left(Y_{i}\right)$ (resp. $\left.l_{1}\left(Y_{i}\right)\right)$ defined by

$$
\mu \circ \varphi_{i}(V)=\mu\left(\varphi_{i}^{-1}(V)\right)
$$

where $V \subset Y_{i}$ is a Borel set.)

If the above is satisfied then $\left\{\varphi_{i}\right\}_{1 \leqslant i \leqslant k}$ is said to be a $\lambda$-measure separating family (resp. a uniformly separating family with constant $\lambda$ ). (This characterization motivated the choice of the terminology "measure separating" and "uniformly separating” family. See $\$ \S 1$ and 2 of [9] for a more detailed study of these concepts. Note

Received by the editors February 12, 1982.

1980 Mathematics Subject Classification. Primary 54F45; Secondary 26B40, 54C30.

(C) 1983 American Mathematical Society $0002-9947 / 82 / 0000-1183 / \$ 04.50$ 
also that by this characterization a measure separating family is also a uniformly separating family and hence if the linear superposition operator $\left(g_{1}, g_{2}, \ldots, g_{k}\right) \rightarrow$ $\sum_{i=1}^{k} g_{i} \circ \varphi_{i}$ maps $C\left(Y_{1}\right) \times C\left(Y_{2}\right) \times \cdots \times C\left(Y_{k}\right)$ onto $C(X)$ then it also maps $l_{\infty}\left(Y_{1}\right)$ $\times \cdots \times l_{\infty}\left(Y_{k}\right)$ onto $l_{\infty}(X)$. It is not clear whether the converse statement is valid. However, for $k=1,2$ it is. (See [9]).)

In this article we shall study the existence and nonexistence of measure separating and uniformly separating families in the case where the spaces $Y_{i}, 1 \leqslant i \leqslant k$, are all of dimension smaller than the dimension of $X$. To begin with, let us mention two results, both related to the case where the functions $\varphi_{i}$ are real valued. The first result is an extension of the well-known superposition theorem of Kolmogorov [4] and of a theorem of Ostrand [8]. We do not state this theorem in its most general version but in a form which suits the purpose of this article. For the general form as well as a proof, see [10].

TheOREM 1. Let $X$ be an $n$-dimensional compact metric space $(n \geqslant 0)$. Then there exists a $1 /(2 n+1)$ measure separating family $\left\{\varphi_{i}\right\}_{i=1}^{2 n+1} \subset C(X)$. Moreover, the set $\left\{\left(\varphi_{1}, \varphi_{2}, \ldots, \varphi_{2 n+1}\right) \in C(X) \times C(X) \times \cdots \times C(X):\left\{\varphi_{i}\right\}_{i=1}^{2 n+1}\right.$ is a $1 /(2 n+1)$ measure separating family\} is residual in $C(X) \times \cdots \times C(X)(2 n+1$ factors $)$, i.e. contains a dense $G_{\delta}$ set.

The second theorem is an inverse of Theorem 1. By now it is known to be valid only for $n \leqslant 6$ [9 and 12], but I strongly believe in its validity for all $n$.

THEOREM 2. Let $X$ be an $n$-dimensional compact metric space $(n \geqslant 2)$. Then no family which consists of $2 n$ real-valued continuous functions on $X$ is uniformly separating.

Note that for $n=1$ Theorem 2 is clearly false.

For a while I had the impression that Theorem 2 might be generalized in the following way: If $X$ is an $n$-dimensional compact metric space, and if $\left\{\varphi_{i}\right\}_{i=}^{k}$ is a uniformly separating family so that $\operatorname{dim} \varphi_{i}(X)<n$ for $1 \leqslant i \leqslant k$, then $\sum_{i=1}^{k} \operatorname{dim} \varphi_{i}(X) \geqslant 2 n+1$. However, after Professor A. Lelek brought to my attention his construction in [6], I was able to construct a measure separating family $\left\{\varphi_{i}\right\}_{i=1}^{4}$ on the square $I^{2}$ so that $\varphi_{i}, 1 \leqslant i \leqslant 3$, are real valued, while $\varphi_{4}$ has a 1 -dimensional range. This construction clearly refutes the above mentioned hypothesis. It turns out that this construction can be carried out in a more general setting which is our main existence theorem.

THEOREM 3. Let $X$ be an $n$-dimensional compact metric space $(n \geqslant 0)$. Then there exist $n$ continuous monotone functions $\left\{\psi_{i}\right\}_{i=1}^{n}$ on $X$, with 1-dimensional range each, and $2 n+1$ functions $\left\{\varphi_{j}\right\}_{j=1}^{2 n+1}$ in $C(X)$, so that for every $0 \leqslant k \leqslant n$ each $k$ of the $\psi_{i}$ 's together with each $2(n-k)+1$ of the $\varphi_{j}$ 's forms a $\lambda_{n, k}$ measure separating family with $\lambda_{n, k}=1 /(2 n+1+2 k(n-k))$. Moreover, the functions $\psi_{i}, 1 \leqslant i \leqslant n$, can be so chosen that the subset $B$ of $[C(X)]^{2 n+1}$ which consists of the elements $\left(\varphi_{1} \cdots \varphi_{2 n+1}\right)$ which satisfy the above, is residual in $[C(X)]^{2 n+1}$. In addition, if $X$ is connected, locally-connected and unicoherent, then $\psi_{i}(X)$ is a dendrite, $1 \leqslant i \leqslant n$. (See §2 for definitions of these concepts.) 
We shall prove Theorem 3 in $\S 3$. In $\S 4$ we shall show that in many cases Theorem 3 is the best possible, i.e. that the numbers $k$ and $2(n-k)+1$ cannot be reduced. This will follow from some slightly more general theorems.

Let us return to the false hypothesis mentioned above. By Theorem 3, with $k=n$, for each compact $n$-dimensional space $X$, there exist a measure separating family $\left\{\varphi_{i}\right\}_{i=1}^{n+1}$ with $\operatorname{dim} \varphi_{i}(X)=1$ (more precisely $\operatorname{dim} \varphi_{i}(X)=1$ for $1 \leqslant i \leqslant n$ and $\varphi_{n+1}$ real valued), i.e. $\Sigma_{i} \operatorname{dim} \varphi_{i}(X)=n+1$, and if $n \geqslant 2$ then $\operatorname{dim} \varphi_{i}(X)<n$. But the functions $\varphi_{i}, 1 \leqslant i \leqslant n$, are not real valued, i.e., the least Euclidean dimension into which $\varphi_{i}(X)$ can be embedded is $\geqslant 2$. So, if we define the Euclidean index $E(W)$ of a separable metric space $W$ to be the smallest integer $m$ such that $W$ is homeomorphic to a subset of $R^{m}$ (of course $\operatorname{dim} W \leqslant E(W) \leqslant 2 \operatorname{dim} W+1$ ) then we obtain $\sum_{i=1}^{n+1} E\left(\varphi_{i}(X)\right)=\sum_{i=1}^{n} E\left(\varphi_{i}(X)\right)+1 \geqslant 2 n+1$. And in the general form of Theorem 1.3

$$
\begin{aligned}
\sum_{i=1}^{k} E\left(\psi_{i}(X)\right)+\sum_{j=1}^{2(n-k)+1} E\left(\varphi_{j}(X)\right) & =\sum_{i=1}^{k} E\left(\psi_{i}(X)\right)+2(n-k)+1 \\
& \geqslant 2 k+2(n-k)+1=2 n+1
\end{aligned}
$$

These facts motivate the following problem, which trivially holds for $n \leqslant 1$, and, as will be shown in $\S 4$, holds also for $n=2$ under some extra assumptions on the space $X$ (e.g. if $\operatorname{dim} X=2$ and $X$ contains $I^{2}$ ).

Problem. Let $X$ be an $n$-dimensional compact metric space, and let $\left\{\varphi_{i}\right\}_{i=1}^{k}$ be a uniformly separating family on $X$, with $\operatorname{dim} \varphi_{i}(X)<n$. Does it follow that $\sum_{i=1}^{k} E\left(\varphi_{i}(X)\right) \geqslant 2 n+1$ ?

2. Preliminaries. In this section we mention some results and concepts that will be used in the following sections.

Definition. (i) Let $\varphi$ and $\psi$ be mappings on a space $X$. $\psi$ is said to be a refinement of $\varphi$ if the cover $\left\{\psi^{-1}(y): y \in \psi(X)\right\}$ of $X$ is a refinement of the cover $\left\{\varphi^{-1}(z): z \in \varphi(X)\right\}$.

(ii) Let $F$ and $G$ be two finite families of mappings on a space $X$. $F$ is said to be a refinement of $G$ if for each $\varphi \in G$ there correspond some $\psi \in F$ so that $\psi$ refines $\varphi$.

The following proposition follows easily from the definitions, and its proof is left to the reader.

2.1. Proposition. Let $X$ be a compact metric space and let $F$ and $G$ be finite families of mappings on $X$ so that $F$ refines $G$. If $G$ is a measure (resp. uniformly) separating family then so if $F$.

2.2. CoRollary. Let $F=\left\{\varphi_{i}\right\}_{i=1}^{k}$ be a measure (resp. uniformly) separating family on a compact metric space $X$. Let $a_{1}, a_{2}, \ldots, a_{m}$ be subsets of $\{1,2, \ldots, k\}$ so that $\bigcup_{j=1}^{m} a_{j}=\{1,2, \ldots, k\}$. Let $\psi_{j}: X \rightarrow \prod_{i \in a_{j}} \varphi_{i}(X)$ be defined by $\psi_{j}(x)=\left(\psi_{i_{1}}(x)\right.$, $\left.\psi_{i_{2}}(x), \ldots, \psi_{i\left|a_{j}\right|}(x)\right)$ where $a_{j}=\left\{i_{1}, i_{2}, \ldots, i_{\left|a_{j}\right|}\right\}$. Then $G=\left\{\psi_{j}\right\}_{j=1}^{m}$ is also a measure (resp. uniformly) separating family. 
2.3. Corollary. Let $F=\left\{\varphi_{i}\right\}_{i=1}^{k}$ be as above. If for each $1 \leqslant i \leqslant k, \varphi_{i}$ factors through some space $Y_{i}$ with factors $\psi_{i}$ and $\tau_{i}$, i.e. there exist spaces $Y_{i}$ and mappings $\psi_{i}$ : $X \rightarrow Y_{i}$ and $\tau_{i}: Y_{i} \rightarrow \varphi_{i}(X)$ so that $\varphi_{i}(x)=\tau_{i}\left(\psi_{i}(x)\right)$, then $H=\left\{\psi_{i}\right\}_{i=1}^{k}$ is also a measure (resp. uniformly) separating family on $X$.

Proof of Corollaries 2.2 AND 2.3. In both cases $G$ and $H$ refine $F$.

Recall that a mapping $\varphi: X \rightarrow Y$ is called monotone if $\varphi^{-1}(K)$ is connected whenever $K \subset Y$ is closed and connected. The dimension of $\varphi$ is defined by $\operatorname{dim} \varphi=\sup _{y \in Y} \operatorname{dim} \varphi^{-1}(y)$. The following are some results related to these concepts.

2.4. Theorem (Hurewicz). If $\varphi: X \rightarrow Y$ is a closed mapping then $\operatorname{dim} X \leqslant \operatorname{dim} Y$ $+\operatorname{dim} \varphi$.

2.5. Theorem (Jung [2], Keesling [3]). If $\varphi: X \rightarrow Y$ is a closed mapping, and $Y^{\prime}=\left\{y \in Y: \operatorname{dim} \varphi^{-1}(y) \geqslant \operatorname{dim} X-\operatorname{dim} Y\right\}$ then $\operatorname{dim} X \leqslant \operatorname{dim} Y^{\prime}+\operatorname{dim} \varphi$.

In both theorems the assumption on $\varphi$ can be replaced by the assumption that $X$ is $\sigma$ compact.

2.6. THEOREM [5, p. 125]. Let $X$ be an n-dimensional space. Then there exists a 0-dimensional mapping $f: X \rightarrow I^{n}$.

2.7. THEOREM [5, p. 184]. Let $X$ be a compact metric space, and $\varphi: X \rightarrow Y a$ mapping. Then $\varphi$ factors through a space $T$ (called the tree of $\varphi$ ) with factors $\psi: X \rightarrow T$ and $\tau: T \rightarrow Y$ so that $\psi$ is monotone, $\operatorname{dim} \tau=0$ and $\varphi(x)=\tau(\psi(x))$.

The elements of $T$ can be identified with the components of the sets $\varphi^{-1}(y)$, $y \in Y, \psi(x)$ is then defined to be the component of $\varphi^{-1}(\varphi(x))$ which contains $x$, and $\tau(t)=\varphi(t)=y$ where $t$ is a component of $\varphi^{-1}(y)$. As $\operatorname{dim} \tau=0$ it follows from Theorem 2.4 that $\operatorname{dim} T \leqslant \operatorname{dim} Y$.

2.8. COROLlaRY. Let $\left\{\varphi_{i}\right\}_{i=1}^{k}$ be a measure (resp. uniformly) separating family on a compact space $X$. Then there exists a measure (resp. uniformly) separating family $\left\{\psi_{i}\right\}_{i=1}^{k}$ on $X$ with $\psi_{i}$ monotone and $\operatorname{dim} \psi_{i}(X) \leqslant \operatorname{dim} \varphi_{j}(X), 1 \leqslant i \leqslant k$.

Proof. Let $\psi_{i}$ be the factor in the factorization of $\varphi_{i}$ as in Theorem 2.7. Then $\psi_{i}$ is monotone, $\operatorname{dim} \psi_{i}(X) \leqslant \operatorname{dim} \varphi_{i}(X)$, and by Corollary $2.3\left\{\psi_{i}\right\}_{i=1}^{k}$ is a measure (resp. uniformly) separating family.

Definition. (i) A compact metric space $D$ which is connected, locally-connected and does not contain a homeomorphic image of the circle is called a dendrite.

(ii) A space $X$ is called unicoherent if $X=X_{1} \cup X_{2}$ with $X_{i} \subset X$, closed and connected, $i=1,2$, implies that $X_{1} \cap X_{2}$ is connected.

The $n$-cube $I^{n}$ is unicoherent for all $n \geqslant 1$. The proofs of the following facts about dendrites can be found in [5, p. 300].

2.9. A dendrite is unicoherent and 1-dimensional.

2.10. A dendrite can be embedded into $R^{2}$.

2.11. A compact connected subset of a dendrite is a dendrite.

2.12. A 1-dimensional connected and locally-connected compact unicoherent metric space is a dendrite. 
2.13. Let $X$ be a compact, connected, locally-connected and unicoherent metric space. If $\psi$ is a monotone mapping on $X$ with $\operatorname{dim} \psi(X)=1$ then $\psi(X)$ is a dendrite.

Definition. Let $X$ be an $n$-dimensional space.

(i) A family $F$ of $n$-dimensional closed subsets of $X$ is said to be a dimensional network for $X$, if every $n$-dimensional closed subset of $X$ contains some element of $F$.

(ii) $X$ is said to be countably $n$-dimensional if it admits a countable dimensional network.

2.14. THEOREM [11]. Let $W$ be a compact subset of $Y_{1} \times Y_{2}$ with $\operatorname{dim} W=\operatorname{dim} Y_{1}+$ $\operatorname{dim} Y_{2}$. If $Y_{i}$ is countably $\operatorname{dim} Y_{i}$-dimensional for $i=1$ or $i=2$, then $W$ contains $a$ product $W \supset Y_{1}^{\prime} \times Y_{2}^{\prime}$ with $\operatorname{dim} Y_{i}^{\prime}=\operatorname{dim} Y_{i}, i=1,2$.

\subsection{TheOREM. A dendrite is countably 1-dimensional.}

Proof. Let $T$ be a dendrite and let $E$ denote the set of end points of $T$, i.e. $E=\{t \in T: T \backslash\{t\}$ is connected $\}$. By a theorem of Menger [7] (see also [1, Theorem 4, p. 139]) $T \backslash E$ can be represented as a countable union of simple arcs $\left\{l_{n}\right\}_{n \geqslant 1}$ which do not intersect pair-wise in more than one point. (Note that $E$ may be uncountable and may be dense in $T$. $T \backslash E$ is always dense in $T$.) For $n \geqslant 1$ let $\left\{l_{n, k}\right\}_{k \geqslant 1}$ be a sequence of simple arcs in $l_{n}$, whose interiors form a basis for the topology of $l_{n}$. We claim that $\left\{l_{n, k}\right\}_{n \geqslant 1, k \geqslant 1}$ is a dimensional network for $T$. Indeed, let $K \subset T$ be closed and 1-dimensional. Then $K$ contains a 1-dimensional component $M$ which by 2.11 is a dendrite too, and hence contains some arc $l$. Let $l^{\prime} \subset l$ be another arc so that $l^{\prime}$ does not contain any of the two end points of $l$. Then each point of $l^{\prime}$ separates $l^{\prime}$ and hence separates $T$, i.e. $l^{\prime} \subset T \backslash E=\cup_{n \geqslant l} l_{n}$. Hence for some $n \geqslant 1, \operatorname{dim}\left(l^{\prime} \cap l_{n}\right)=1$ and thus $l^{\prime} \cap l_{n}$ contains some arc, and also one of the arcs $l_{n, k}$ for some $k \geqslant 1$, and the theorem follows.

\section{Proof of Theorem 3.}

3.1. The construction of Lelek. In [6] A. Lelek constructs a mapping $\varphi$ of $I^{n}$ onto a dendrite $T$ with some remarkable properties. In particular $D_{\varphi}=\left\{x \in I^{n}:\{x\}=\right.$ $\left.\varphi^{-1}(\varphi(x))\right\}$ is a dense $G_{\delta}$ in $I^{n}$, and $E_{\varphi}=I^{n} \backslash D_{\varphi}$ has a simple explicit description. This mapping $\varphi$ will play an important role in the proof of Theorem 3. We shall describe the construction below, giving detailed attention only to parts which will be relevant to our needs. The others can be found in [6]. Actually, we shall describe an upper semicontinuous decomposition $L$ of $I^{n}$ so that $\varphi$ is the mapping induced by $L$, i.e. the elements of $L$ are the fibers of $\varphi$.

Let $\Delta$ denote the classical Cantor set in $[0,1]$, and let $P$ be an $n$-cube in $R^{n}$ with boundary $\partial P$ and center $q$. (At this point we are more restrictive than in the original construction [6]. There $P$ could be any $n$-dimensional parallelepiped. The restriction to cubes will turn out to be more convenient later. Note that by an $n$-cube we mean any similar copy of the interior of $I^{n}$.) $R^{n}$ will be looked upon as a vector space over $R$. Set $A=\{t x+(1-t) q: t \in \Delta \neq 1, x \in \partial P\}$.

$A$ is a nowhere dense closed subset of $P, A \cup \partial P$ is compact and $\operatorname{dim}(A \cup \partial P)=$ $n-1$. $P \backslash A$ consists of countably many components, each of which is a domain $D$ in $R^{n}$ with boundary of the form $\partial D=\left\{t_{i} x+\left(1-t_{i}\right) q: i=1,2, x \in \partial P\right\}$ where $t_{1}$ 
and $t_{2}$ are end points of some component interval of $[0,1] \backslash \Delta$, i.e. $t_{1}=k / 3^{m}$ and $t_{2}=(k+1) / 3^{m}$ for some $m \geqslant 1$ and $1 \leqslant k \leqslant 3^{m}-1$. Let us cut each of these domains $D$ with finitely many compact pieces of $(n-1)$-dimensional hyperplanes contained in $\bar{D}$ to obtain finitely many congruent cubes $P^{\prime}$, so that each $P^{\prime}$ is of the form $P^{\prime}=a+\alpha P$ where $a \in R^{n}$ and $0<\alpha<1$. (Actually if $\partial D$ has the above representation with $t_{1}=k / 3^{m}$ and $t_{2}=(k+1) / 3^{m}$ then $\alpha=1 / 3^{m}$. Note that here again we are more restrictive than in [6].) Let $A^{\prime}$ denote the union of $A$ and of all these $(n-1)$-dimensional pieces, where $D$ ranges over all the components of $P \backslash A$. (Note that there are countably many such pieces.) Let $L_{1}$ denote the family of components of $A^{\prime}$. $A_{1}$ contains elements of two major types: components of the form $\{t x+(1-t) q: x \in \partial P\}$ with $1 \neq t \in \Delta$ and $t$ not of the form $k / 3^{m}$, and components of the form

$$
\begin{aligned}
\left\{k / 3^{m} x+\left(1-k / 3^{m}\right) q: x \in \partial P\right\} & \\
\cup & \left.\left\{(k+1) / 3^{m}\right) x+\left(1-(k+1) / 3^{m}\right) q: x \in \partial P\right\} \\
& \cup\{\text { finitely many }(n-1) \text {-dimensional pieces }\}
\end{aligned}
$$

Now we continue the construction as follows: The components of $P \backslash A^{\prime}$ consists of countably many $n$-cubes of the form $a+\alpha P$. On each of these $n$-cubes we operate in the same way as we operated on $P$. In this way we obtain in each of these $n$-cubes sets similar to $A$ and $A^{\prime}$. The collection of components of $P \backslash\{$ the union of all the $A$ 's (including the original $A$ ) $\}$ consists again of a countable family of $n$-cubes, and we continue by an obvious deduction. In this way we obtain countable collection of sets $\left\{A_{l}\right\}_{l=1}^{\infty}$ and $\left\{A_{l}^{\prime}\right\}_{l=1}^{\infty}$ where for each $l \geqslant 1, A_{l}^{\prime}$ is of the form $A_{l}^{\prime}=b_{l}+\beta_{l} A^{\prime}$ $\left(b_{l} \in R^{n}, 0<\beta_{l}<1\right)$. Let us assume also that $A^{\prime}=A_{1}^{\prime}$. Let $L_{l}$ denote the family of components of $A_{l}^{\prime}$, and set

$$
L=\bigcup_{l=1}^{\infty} L_{l} \cup\{\partial P\} \cup\left\{\text { the singletons of } P \backslash \bigcup_{l=1}^{\infty} A_{l}^{\prime}\right\} .
$$

Then $L$ is a decomposition of $\bar{P}$. The reader may prove (or else check in [6]) that $L$ is an upper semicontinuous decomposition of $\bar{P}$. Hence the quotient map $\varphi$ induced by $L$ maps $\bar{P}$ onto a compact metric space $T$. It is easy to check (see [6]) that $\operatorname{dim} T=1$, and clearly the elements of $L$ are connected, i.e., $\varphi$ is a monotone mapping. Hence by $2.13 T$ is a dendrite. (Recall that $\bar{P}$ is homeomorphic to $I^{n}$ and hence is locally-connected and unicoherent.) The sets $\overline{A_{l}^{\prime}}$ are nowhere dense in $\bar{P}$, and hence, by the Bair category theorem $P \backslash \cup_{l=1}^{\infty} A_{l}$ is a dense $G_{\delta}$. Since obviously $D_{\varphi}=\{x \in$ $\left.\bar{P}:\{x\}=\varphi^{-1}(\varphi(x))\right\}=P \backslash \cup_{l=1}^{\infty} A_{l}, D_{\varphi}$ is a dense $G_{\delta}$ in $P$. Note also that $\cup_{l=1}^{\infty} \overline{A_{l}^{\prime}}$ $=\cup_{l=1}^{\infty} \mathrm{A}_{l}^{\prime} \cup \partial P$, and that $E_{\varphi}=\bar{P} \backslash D_{\varphi}=\cup_{l=1}^{\infty} \overline{A_{l}^{\prime}}$. This completes the construction of $\varphi$.

We shall apply now the construction 3.1 to prove the following lemma.

3.2. Lemma. Let $X=I^{n}(n \geqslant 2)$. There exist $n$ monotone mappings $\left\{\varphi_{i}\right\}_{i=1}^{n}$ on $X$, such that $\varphi_{i}$ maps $X$ onto a dendrite, and so that for each $1 \leqslant k \leqslant n$ and $1 \leqslant i_{1}<i_{2}<$ $\cdots<i_{k} \leqslant n, \operatorname{dim} \bigcap_{l=1}^{k} E_{i_{l}} \leqslant n-k$, where $E_{i}=X \backslash D_{i}$ and $D_{i}=\{x \in X:\{x\}=$ $\left.\varphi_{i}^{-1}\left(\varphi_{i}(x)\right)\right\}$. (Note that $E_{i}$ is an $F_{\sigma}$ in $X$.) 
Before giving the detailed proof, we shall describe intuitively the case $n=2$ which exhibits the basic ideas. Let $P_{1}=\left\{(x, y) \in R^{2}:|x|<1,|y|<1\right\}$ be the canonical square, and let $\varphi_{1}: \bar{P}_{1} \rightarrow T$ be the mapping associated to $P_{1}$ by the construction 3.1. Let $P_{2}$ be the square obtained from $P_{1}$ by rotation by $45^{\circ}$ about the origin, and let $\varphi_{2}: \overline{P_{2}} \rightarrow T$ be the mapping associated to $P_{2}$ by 3.1. Set $X=\overline{P_{1}} \cap \overline{P_{2}}$. Obviously, $X$ is homeomorphic to $I^{2}$, and we claim that the restriction to $X$ of $\varphi_{1}$ and $\varphi_{2}$ satisfy the conditions of the lemma. For $k=1$ this follows directly from 3.1. For $k=2$ this is less obvious but still true: as mentioned in $3.1 E_{1} \subset \cup_{l=1}^{\infty} \overline{A_{1, l}^{\prime}}$ where the $A_{1, l}^{\prime}$ are the sets constructed in 3.1 with $P=P_{1}$. Respectively $E_{2} \subset \cup_{l=1}^{\infty} \overline{A_{2, l}^{\prime}}$. Hence, to prove $\operatorname{dim}\left(E_{1} \cap E_{2}\right) \leqslant 0$ it suffices to show that $\operatorname{dim}\left(\overline{A_{1, l_{1}}^{\prime}} \cap \overline{A_{2, l_{2}}^{\prime}}\right) \leqslant 0$ for all $l_{1}$ and $l_{2}$. But this is intuitively clear: $\overline{A_{1, l_{1}}^{\prime}}$ is the set constructed in 3.1 , and $\overline{A_{2, l_{2}}^{\prime}}$ is obtained from $\overline{A_{1, l_{1}}^{\prime}}$ by rotation by $45^{\circ}$, and a possible translation and multiplication by a scalar. Thus the intersection does not contain any straight line segment, and hence is zero-dimensional.

The following proof uses the same ideas.

Proof of LemMA 3.2. Let $n \geqslant 2$, and let $\left\{e_{i, j}\right\}_{1 \leqslant i, j \leqslant n}$ be $n^{2}$ vectors in $R^{n}$ so that:

(i) For $1 \leqslant i \leqslant n,\left\{e_{i, j}\right\}_{j=1}^{n}$ is an orthonormal basis for $R^{n}$.

(ii) $\operatorname{dim} \cap_{l=1}^{k} H_{i_{l}, j_{l}}=n-k$ for $1 \leqslant k \leqslant n, 1 \leqslant i<i_{2}<\cdots<i_{k} \leqslant n$, and $1 \leqslant j_{l}$ $\leqslant n$ where $H_{i, j}$ is the subspace orthogonal to $e_{i, j}$.

For $1 \leqslant i \leqslant n$, let $P_{i}$ be the canonical $n$-cube with center at the origin and volume $2^{n}$ whose sides are contained in the hyperplanes $\pm e_{i, j}+H_{i, j}, 1 \leqslant j \leqslant n$. Set $X=$ $\bigcap_{i=1}^{n} \bar{P}_{i}$. Then $X$ is a convex body in $R^{n}$ and hence homeomorphic to $I^{n}$. For $1 \leqslant i \leqslant n$, let $\varphi_{i}$ be the mapping associated to $P_{i}$ by the construction 3.1. We claim that the restrictions of the mappings $\varphi_{i}$ to $X$ satisfy the conditions of the lemma.

For $1 \leqslant i, j \leqslant n$ set $H_{i, j}^{\prime}=e_{i, j}+H_{i, j}=\left\{e_{i, j}+x: x \in H_{i, j}\right\}$.

Then there exists a 0 -dimensional $F_{\sigma}$ subset $V$ of $[-1,1]$ so that $E_{i} \subset \cup_{j=1}^{n} V \cdot H_{i, j}^{\prime}$, for all $1 \leqslant i \leqslant n$ (where $V \cdot H=\{t \cdot x: t \in V, x \in H\}$ ). To see this, fix some $1 \leqslant i \leqslant n$. In the following we shall omit the index $i$. Let $A$ be the set defined in the construction 3.1. Then $\bar{A}=\Delta \cdot \partial P$. Now $\partial P$ is contained in the union of the hyperplanes $\pm H_{j}^{\prime}, 1 \leqslant j \leqslant n$. So $\bar{A} \subset \cup_{j=1}^{n}(\Delta \cup(-\Delta)) \cdot H_{j}^{\prime}$. The set $\overline{A^{\prime}}$ has been obtained from $\vec{A}$ by adding to it countably many compact pieces of hyperplanes of the form $t \cdot H_{j}^{\prime}, 1 \leqslant j \leqslant n,-1 \leqslant t \leqslant 1$. Let $\tau$ denote the set of all those numbers $t$. Then

$$
\overline{A^{\prime}} \subset \bigcup_{j=1}^{n}(\Delta \cup(-\Delta) \cup \tau) \cdot H_{j}^{\prime}=\bigcup_{j=1}^{n} V_{1} \cdot H_{j}^{\prime},
$$

where $V_{1}=\Delta \cup(-\Delta) \cup \tau$. Clearly $V_{1}$ is a 0 -dimensional $F_{\sigma}$ in $[-1,1]$. For a fixed $l \geqslant 1, \overline{A_{l}^{\prime}}=b_{l}+\beta_{l} \overline{A^{\prime}}, b_{l} \in R^{n}, 0 \leqslant \beta_{l} \leqslant 1$. Let $b_{l}=\left(b_{l, 1}, \ldots, b_{l, n}\right)$ be the coordinates of $b_{l}$ with respect to the basis $\left\{e_{j}\right\}_{j=1}^{n}$. Then

$$
\begin{aligned}
\overline{A_{l}^{\prime}} & =b_{l}+\beta_{l} \overline{A^{\prime}} \subset b_{l}+\beta_{l} \bigcup_{j=1}^{n} V_{1} \cdot H_{j}^{\prime}=b_{l}+\bigcup_{j=1}^{n} \beta_{l} V_{1} \cdot H_{j}^{\prime} \\
& =\bigcup_{j=1}^{n}\left(b_{l}+\beta_{l} V_{l} \cdot H_{j}^{\prime}\right)=\bigcup_{j=1}^{n}\left(b_{l, j}+\beta_{l} V_{1}\right) \cdot H_{j}^{\prime} .
\end{aligned}
$$


The last equality holds since $H_{j}^{\prime}=H_{j}+e_{j}$ and $H_{j}$ is the orthogonal subspace of $e_{j}$. Hence

$$
\begin{aligned}
b_{l}+\beta_{l} V_{1} H_{j}^{\prime} & =b_{l}+\beta_{l} V_{1}\left(e_{j}+H_{j}\right)=b_{l}+\beta_{l} V_{1} e_{j}+H_{j} \\
& =\left(b_{l, j}+\beta_{l} V_{1}\right) e_{j}+b_{l}-b_{l, j} e_{j}+H_{j} \\
& =\left(b_{l, j}+\beta_{l} V_{1}\right) e_{j}+H_{j} \quad\left(\text { since } b_{l}-b_{l, j} e_{j} \in H_{j}\right) \\
& =\left(b_{l, j}+\beta_{l} V_{1}\right)\left(e_{j}+H_{j}\right)=\left(b_{l, j}+\beta_{l} V_{1}\right) \cdot H_{j}^{\prime} .
\end{aligned}
$$

So, if we let $V_{l, j}=b_{l, j}+\beta_{l} V_{1}$, and $V_{l}=\cup_{j=1}^{n} V_{l, j}$ then $\overline{A_{l}^{\prime}} \subset \cup_{j=1}^{n} V_{l, j} \cdot H_{j}^{\prime} \subset$ $\cup_{j=1}^{n} V_{l} \cdot H_{j}^{\prime}$, and $V_{l, j}$ as well as $V_{l}$ are 0 -dimensional $F_{\sigma}$ 's in $[-1,1]$. Hence

$$
E=\bigcup_{l=1}^{\infty} \overline{A_{l}^{\prime}} \subset \bigcup_{l=1}^{\infty} \bigcup_{j=1}^{n} V_{l} \cdot H_{j}^{\prime} \subset \bigcup_{j=1}^{n} V \cdot H_{j}^{\prime}
$$

where $V=\cup_{l=1}^{\infty} V_{l}$ is a 0 -dimensional $F_{\sigma}$ in $[-1,1]$.

We shall now prove the lemma with $\left\{i_{1}, i_{2}, \ldots, i_{k}\right\}=\{1,2, \ldots, k\}$. By the above $\bigcap_{i=1}^{k} E_{i} \subset F=\bigcap_{i=1}^{n} \cup_{j=1}^{n} V \cdot H_{i, j}^{\prime}$. Hence, it suffices to show that $\operatorname{dim} F \leqslant n-k$. But $F$ can be also represented as $F=\cup \cap_{i=1}^{k} V \cdot H_{i, j}^{\prime}$ where the union is taken over all the elements $\left(j_{1}, j_{2}, \ldots, j_{k}\right)$ of $\{1,2, \ldots, n\}^{k}$, and since each of these united sets is $\sigma$ compact it suffices to show that $\operatorname{dim}\left(\bigcap_{i=1}^{k} V \cdot H_{i, j_{i}}^{\prime}\right) \leqslant n-k$ for each $\left(j_{1}, j_{2}, \ldots, j_{k}\right)$. So fix $\left(j_{1}, \ldots, j_{k}\right)$. Define a mapping $f: \bigcap_{i=1}^{k} V \cdot H_{i, j_{i}}^{\prime} \rightarrow V^{k}$ by $f(x)=\left(t_{1}, \ldots, t_{k}\right)$ if and only if $x \in \cap_{i=1}^{k}\left(t_{i} H_{i, j_{i}}^{\prime}\right) . f$ is well defined since $\left(t_{1}, \ldots, t_{k}\right) \neq\left(t_{1}^{\prime}, \ldots, t_{k}^{\prime}\right)$ implies

$$
\bigcap_{i=1}^{k}\left(t_{i} H_{i, j_{i}}^{\prime}\right) \cap \bigcap_{i=1}^{k}\left(t_{i}^{\prime} H_{i, j_{i}}^{\prime}\right)=\varnothing .
$$

(Actually $t_{i} \neq t_{i}^{\prime}$ implies $t_{i} \cdot H_{i, j_{i}}^{\prime} \cap t_{i}^{\prime} \cdot H_{i, j_{i}}^{\prime}=\varnothing$.) $f$ is continuous, and by (ii)

$$
\operatorname{dim} f^{-1}\left(t_{1}, \ldots, t_{k}\right)=\operatorname{dim}\left(\bigcap_{i=1}^{k} t_{i} \cdot H_{i, j_{i}}^{\prime}\right) \leqslant n-k,
$$

for all $\left(t_{1}, \ldots, t_{k}\right) \in V^{k}$, i.e. $\operatorname{dim} f \leqslant n-k$. As $\cap_{i=1}^{k} V \cdot H_{i, j_{i}}^{\prime}$ is $\sigma$ compact, it follows from Theorem 2.4 that

$$
\operatorname{dim} \bigcap_{i=1}^{k} V \cdot H_{i, j_{i}}^{\prime} \leqslant \operatorname{dim} V^{k}+\operatorname{dim} f \leqslant n-k
$$

since $\operatorname{dim} V^{k}=0$. This completes the proof of the lemma.

The next lemma is a generalization of Lemma 3.2 to general compact metric spaces.

3.3. LEMMA. Let $X$ be an $n$-dimensional compact metric space $(n \geqslant 2)$. Then there exist monotone mappings $\psi_{i}: X \rightarrow S_{i}, 1 \leqslant i \leqslant n$, so that $\operatorname{dim} S_{i}=1$, and such that for each $1 \leqslant k \leqslant n, \operatorname{dim} \cap_{l=1}^{k} F_{i_{l}} \leqslant n-k$, where $F_{i}=X \backslash D_{i}$ and $D_{i}=\{x \in X:\{x\}=$ $\left.\psi_{i}^{-1}\left(\psi_{i}(x)\right)\right\}$.

Proof. Let $X$ be an $n$-dimensional compact metric space. Then (Theorem 2.6) $X$ admits a 0-dimensional mapping $f: X \rightarrow I^{n}$. Let $\varphi_{i}: I^{n} \rightarrow T$ be the mappings guaranteed by Lemma 3.2. Let $S_{i}$ be the tree of the mapping $\varphi_{i} \circ f: X \rightarrow T$ and let 
$\psi_{i}: X \rightarrow S_{i}$ and $\tau_{i}: S_{i} \rightarrow T$ be the mappings so that $\varphi_{i} \circ f=\tau_{i} \circ \psi_{i}$ as in Theorem 2.7. Recali that the elements of $S_{i}$ are components of fibers of $\varphi_{i} \circ f . \psi_{i}(x)$ is the component which contains $x$, and $\tau_{i}(s)=\varphi_{i}(f(s)), s \in S_{i}$ :

$$
\begin{array}{ccc}
X & \stackrel{\psi_{i}}{\rightarrow} & S_{i} \\
f \downarrow & \rightarrow & \downarrow \tau_{i} \\
I^{n} & \vec{\varphi}_{i} & T
\end{array}
$$

We claim that the $\left\{\psi_{i}\right\}_{i=1}^{n}$ do the job. Note first that by Theorem $2.7 \operatorname{dim} S_{i}=1$, and the functions $\psi_{i}$ are monotone. Also, $F_{i} \subset f^{-1}\left(E_{i}\right)$ where $E_{i}$ are the subsets of $I^{n}$ associated to $\varphi_{i}$ in Lemma 3.2. Indeed, let $x \in F_{i}$. Hence there exists some $x^{\prime} \neq x$ so that $\psi_{i}(x)=\psi_{i}\left(x^{\prime}\right)=s \in S_{i}$. Set $\tau_{i}(s)=t \in T$. Then $s$ is a component of $\left(\varphi_{i} \circ f\right)^{-1}(t)$, because $\left(\varphi_{i} \circ f\right)(x)=\tau_{i}\left(\psi_{i}(x)\right)=\tau_{i}(s)=t$ and hence $x \in f^{-1}\left(\varphi_{i}^{-1}(t)\right)$, and $s$ is the component of the fiber of $\varphi_{i} \circ f$ which contains $x$. So, in particular $s \subset f^{-1}\left(\varphi_{i}^{-1}(t)\right)$, and also $f(s) \subset \varphi_{i}^{-1}(t)$. But $s$ is a compact connected set which contains more than one point $\left(x, x^{\prime} \in s\right)$ hence $\operatorname{dim} s \geqslant 1$, and since $\operatorname{dim} f=0$, $\operatorname{dim} f(s) \geqslant 1$ too, and in particular $f(s)$ contains more than one point. Thus, $f(s)$ is contained in $\varphi_{i}^{-1}(t)$, and is not a singleton, which means that $f(s) \subset E_{i}$, and $x \in S \subset f^{-1}(f(s)) \subset f^{-1}\left(E_{i}\right)$.

It follows that $\bigcap_{i=1}^{k} F_{i} \subset \cap_{i=1}^{k} f^{-1}\left(E_{i}\right)=f^{-1}\left(\cap_{i=1}^{k} E_{i}\right)$. But by Lemma 3.2 $\operatorname{dim} \cap_{i=1}^{k} E_{i} \leqslant n-k$, and since $\bigcap_{i=1}^{k} E_{i}$ is an $F_{\sigma}$ and $\operatorname{dim} f=0$, it follows from the Hurewicz Theorem 2.4 that $\operatorname{dim} f^{-1}\left(\cap_{i=1}^{k} E_{i}\right) \leqslant \operatorname{dim} \bigcap_{i=1}^{k} E_{i}+\operatorname{dim} f \leqslant n-k$, and the lemma follows.

3.4. Lemma. Let $X$ be a compact metric space, and let $E \subset X$ be an $F_{\sigma}$ with $\operatorname{dim} E \leqslant m \quad(m \geqslant 0)$. Then the subset $A$ of $[C(X)]^{2 m+1}$ defined by $A=$ $\left\{\left(\varphi_{1}, \varphi_{2}, \ldots, \varphi_{2 m+1}\right) \in[C(X)]^{2 m+1}:\left\{\varphi_{i}\right\}_{i=1}^{2 m+1}\right.$ is a $1 /(2 m+1)$ measure separating family on $E\}$ is residual in $[C(X)]^{2 m+1}$.

REMARK. The concept of a $\lambda$ measure separating family has been defined on compact spaces. By a " $\lambda$ measure separating family on $E$ " we mean that for every $\mu \in M(X)$ so that the support of $\mu$ is contained in $E$, there correspond some $\varphi_{i}$ so that $\left\|\mu \circ \varphi_{i}\right\| \geqslant \lambda\|\mu\|$.

Proof. Since $E$ is an $F_{\sigma}$, it has a representation $E=\cup_{l=1}^{\infty} E_{l}$ with $E_{l} \subset E_{l+1}$, and $E_{l}$ compact and $\operatorname{dim} E_{l} \leqslant m$. Set

$$
\begin{aligned}
A_{l}=\left\{\left(\varphi_{1}, \ldots, \varphi_{2 m+1}\right)\right. & \in[C(X)]^{2 m+1}: \\
& \left.\left\{\varphi_{i}\right\}_{i=1}^{2 m+1} \text { is a } \frac{1}{2 m+1} \text { measure separating family on } E_{l}\right\}
\end{aligned}
$$

and

$$
\begin{aligned}
& A_{l}^{\prime}=\left\{\left(\psi_{1}, \ldots, \psi_{2 m+1}\right) \in\left[C\left(E_{l}\right)\right]^{2 m+1}:\right. \\
& \left.\quad\left\{\psi_{i}\right\}_{i=1}^{2 m+1} \text { is a } \frac{1}{2 m+1} \text { measure separating family on } E_{l}\right\} .
\end{aligned}
$$


By Theorem 1, $A_{l}^{\prime}$ is residual in $\left[C\left(E_{l}\right)\right]^{2 m+1}$, and hence $A_{l}^{\prime} \supset \bigcap_{k=1}^{\infty} U_{k}^{\prime}$ where $U_{k}^{\prime}$ are open and dense in $\left[C\left(E_{l}\right)\right]^{2 m+1}$. Obviously, if $\left(\psi_{1}, \ldots, \psi_{2 m+1}\right) \in A_{l}^{\prime}$, and $\left(\varphi_{1}, \ldots, \varphi_{2 m+1}\right) \in[C(X)]^{2 m+1}$ is such that $\varphi_{i} / E_{l}=\psi_{i}, \quad 1 \leqslant i \leqslant 2 m+1$, then $\left(\varphi_{1}, \ldots, \varphi_{2 m+1}\right) \in A_{l}$. It follows that $A_{l} \supset \cap_{k=1}^{\infty} U_{k}=\left\{\left(\varphi_{1}, \ldots, \varphi_{2 m+1}\right)\right.$ : $\left.\left(\varphi_{1 / E_{1}}, \ldots, \varphi_{2 m+1 / E_{l}}\right) \in U_{k}^{\prime}\right\}$. We claim that $U_{k}$ is open and dense in $[C(X)]^{2 m+1}$. Let $\varphi=\left(\varphi_{1}, \ldots, \varphi_{2 m+1}\right) \in U_{k}$. Then $\varphi / E_{l}=\left(\varphi_{1 / E_{l}}, \ldots, \varphi_{2 m+1 / E_{l}}\right) \in U_{k}^{\prime}$. Hence, since $U_{k}^{\prime}$ is open, there exists some $\varepsilon>0$ so that $\psi \in\left[C\left(E_{l}\right)\right]^{2 m+1}$ and $\left\|\psi-\varphi_{/ E_{l}}\right\|_{E_{l}}$ $<\varepsilon$ implies $\psi \in U_{k}^{\prime}$. (The norm in $[C(X)]^{2 m+1}$ is $\|\varphi\|=\max _{1 \leqslant i<2 m+1}\left\|\varphi_{i}\right\|$, where $\|\cdot\|$ is the sup norm in $C(X)$.) It follows that if $\tau=\left(\tau_{1}, \ldots, \tau_{2 m+1}\right) \in[C(X)]^{2 m+1}$ and $\|\tau-\varphi\|_{x}<\varepsilon$, then in particular $\left\|\tau / E_{l}-\varphi / E_{l}\right\|_{E_{l}}<\varepsilon$ and hence $\tau / E_{l} \in U_{k}^{\prime}$, i.e. $\tau \in U_{k}$, and thus $U_{k}$ is open. To see that the $U_{k}$ is dense, let $\tau \in[C(X)]^{2 m+1}$ and $\varepsilon>0 . U_{k}^{\prime}$ is dense in $\left[C\left(E_{l}\right)\right]^{2 m+1}$ hence there exists some $\psi \in U_{k}^{\prime}$ so that $\left\|\tau / E_{l}-\psi\right\|_{E_{l}}<\varepsilon$. Applying Tietze's extension theorem we can extend $\tau_{i}-\psi_{i} / E_{l}$ to an element $f_{i} \in C(X)$ so that $\left\|f_{i}\right\|<\varepsilon, 1 \leqslant i \leqslant 2 m+1$. Set $\hat{\psi}_{i}=f_{i}+\tau_{i} \in C(X)$. Then $\left\|\hat{\psi}_{i}-\tau_{i}\right\|=\left\|f_{i}\right\|<\varepsilon$ and $\hat{\psi}_{i} / E_{l}=\psi_{i}-\tau_{i} / E_{l}+\tau_{i} / E_{l}=\psi_{i}$, i.e. $\hat{\psi}_{i}$ is an extension of $\psi_{i}$, and hence $\hat{\psi}=\left(\hat{\psi}_{1}, \ldots, \hat{\psi}_{2 m+1}\right) \in U_{k}$, and it follows that $U_{k}$ is dense in $[C(X)]^{2 m+1}$. Hence $A_{l}$ is residual in $[C(X)]^{2 m+1}$ since it contains $\cap_{k=1}^{\infty} U_{k}$, and $\cap_{l=1}^{\infty} A_{l}$ is residual too. We claim that $A=\cap_{l=1}^{\infty} A_{l}$. Clearly, if $\varphi \in A$ then $\varphi \in$ $\cap_{l=1}^{\infty} A_{l}$. Let $\varphi \in \cap_{l=1}^{\infty} A_{l}$, and let $\mu$ be an element of $M(X)$ with $\|\mu\|=1$ and $\operatorname{supp} \mu \subset E$. Let $\varepsilon>0$. By the regularity of the measure $\mu$, there exists some $l \geqslant 1$ so that $|\mu|\left(E_{l}\right) \geqslant 1-\varepsilon$ (where $|\mu|$ is the variation of $\mu$ ). Hence $\left\|\mu / E_{l}\right\| \geqslant 1-\varepsilon$ and $\left\|\mu /{ }_{X \backslash E_{l}}\right\| \leqslant \varepsilon$. $\varphi=\left(\varphi_{1}, \ldots, \varphi_{2 m+1}\right) \in A_{l}$, hence, for some $1 \leqslant i \leqslant 2 m+1$, $\left\|\left(\mu / E_{l}\right) \circ \varphi\right\| \geqslant 1 /(2 m+1) \cdot(1-\varepsilon)$ and since $\left\|\mu / X_{X \backslash E_{l}}\right\| \leqslant \varepsilon$ we conclude that $\left\|\mu \circ \varphi_{i}\right\| \geqslant 1 /(2 m+1)(1-\varepsilon)-\varepsilon$, and since $\varepsilon$ was arbitrary it follows that $\left\|\mu \circ \varphi_{i}\right\|$ $\geqslant 1 /(2 m+1)$ for some $1 \leqslant i \leqslant 2 m+1$, i.e. $\varphi \in A$. This proves the lemma.

Proof of TheOrem 3. Let $X$ be an $n$-dimensional compact metric space. The theorem is trivial if $n=0$ (take $\varphi$ to be an embedding of $X$ into $R$ ) and also if $n=1$. (Take $\left\{\varphi_{j}\right\}_{j=1}^{3} \subset C(X)$ a $1 / 3$ measure separating family (by Theorem 1), and let $\psi$ : $X \rightarrow X$ be the identity mapping.) So assume $n \geqslant 2$. Let $\left\{\psi_{i}\right\}_{i=1}^{n}$ be the mappings of Lemma 3.3. For $\left(i_{1}, i_{2}, \ldots, i_{k}\right)=\alpha$ where $1 \leqslant i_{1}<i_{2}<\cdots<i_{k} \leqslant n$, let $F_{\alpha}=$ $F_{\left(i_{1}, \ldots, i_{k}\right)}=\cap_{j=1}^{k} F_{i_{j}}$ where $F_{i}, 1 \leqslant i \leqslant n$, are the sets defined in Lemma 3.3. Recall that by this lemma $F_{\alpha}$ is an $(n-k)$-dimensional $F_{\sigma}$ in $X$. Set

$$
\begin{aligned}
& B_{0}=\left\{\left(\varphi_{1}, \varphi_{2}, \ldots, \varphi_{2 n+1}\right) \in[C(X)]^{2 n=1}:\right. \\
&\left.\{\varphi\}_{j=1}^{2 n+1} \text { is a } \frac{1}{2 n+1} \text { measure separating family on } X\right\}
\end{aligned}
$$

and for $\alpha=\left(i_{1}, \ldots, i_{k}\right)$ as above let $A_{\alpha}$ be the subset of $[C(X)]^{2(n-k)+1}$ defined by

$$
\begin{aligned}
A_{\alpha}=\left\{\left(\varphi_{1}, \ldots, \varphi_{2(n-k)+1}\right):\right. & \\
& \left.\quad\left\{\varphi_{j}\right\}_{j=1}^{2(n-k)+1} \text { is a } \frac{1}{2(n-k)+1} \text { measure separating family on } F_{\alpha}\right\} .
\end{aligned}
$$

By Lemma $3.4 A_{\alpha}$ is residual in $[C(X)]^{2(n-k)+1}$ and so is $B_{0}$ in $[C(X)]^{2 n+1}$. 
For $\beta=\left(j_{1}, j_{2}, \ldots, j_{2(n-k)+1}\right)$ with $1 \leqslant j_{1}<j_{2}<\cdots<j_{2(n-k)+1} \leqslant 2 n+1$, let $B_{\alpha, \beta}$ be the subset of $[C(X)]^{2 n+1}$ defined by $B_{\alpha, \beta}=\left\{\left(\varphi_{1}, \ldots, \varphi_{2 n+1}\right):\left\{\varphi_{j}\right\}_{j \in \beta}\right.$ is in $\left.A_{\alpha}\right\}$. (Note that $|\beta|=2(n-|\alpha|)+1$ where $|\cdot|$ stands for cardinality.)

Then $B_{\alpha, \beta}$ is residual in $[C(X)]^{2 n+1}$ since $B_{\alpha, \beta}$ can be identified with the Cartesian product of $A_{\alpha} \subset[C(X)]^{2(n-k)+1}$ and $[C(X)]^{2 k}$. It follows that

$$
\begin{aligned}
& B_{\alpha}=\bigcap_{\beta} B_{\alpha, \beta}=\left\{\left(\varphi_{1}, \ldots, \varphi_{2 n+1}\right) \in[C(X)]^{2 n+1}:\right. \\
& \text { for all } 1 \leqslant j_{1}<\cdots<j_{2(n-k)+1} \leqslant 2 n+1,\text { measure separating family on } \left.F_{\alpha}\right\} \\
&\left\{\varphi_{j_{l}}\right\}_{l=1}^{2(n-k)+1} \text { is a } \frac{1}{2(n-k)+1}
\end{aligned}
$$

is residual in $[C(X)]^{2 n+1}$, and thus the set $B=\bigcap_{\alpha} B_{\alpha} \cap B_{0}$ is residual in $[C(X)]^{2 n+1}$ too.

We shall see now that each element $\left(\varphi_{1}, \ldots, \varphi_{2 n+1}\right)$ of $B$ satisfies Theorem 3 (with respect to $\left\{\psi_{i}\right\}_{i=1}^{n}$ of course). Let $k \psi$ 's and $(2(n-k)+1) \varphi$ 's be given. Without loss of generality we may assume that these are $\left\{\psi_{i}\right\}_{i=1}^{k}$ and $\left\{\varphi_{j}\right\}_{j=1}^{2(n-k)+1}$. Let $\alpha=(1,2, \ldots, k)$. Then $\left(\varphi_{1}, \ldots, \varphi_{2 n+1}\right) \in B_{\alpha}$ and hence $\left(\varphi_{1}, \ldots, \varphi_{2(n-k)+1}\right) \in A_{\alpha}$, i.e. $\left\{\varphi_{j}\right\}_{j=1}^{2(n-k)+1}$ is a $1 /(2(n-k)+1)$ measure separating family on $F_{\alpha}=\bigcap_{i=1}^{k} F_{i}$. Recall also that $D_{i}=X \backslash F_{i}=\left\{x \in X:\{x\}=\psi_{i}^{-1}\left(\psi_{i}(x)\right)\right\}$ and that $\psi_{i}\left(F_{i}\right) \cap \psi_{i}\left(D_{i}\right)$ $=\varnothing, 1 \leqslant i \leqslant n$. Let $\mu \in M(X)$ be of norm 1 , and let $\delta$ be real with $0 \leqslant \delta \leqslant 1$. Consider the two possible cases

(i) $|\mu|\left(F_{\alpha}\right)<1-\delta$, and

(ii) $|\mu|\left(F_{\alpha}\right) \geqslant 1-\delta$.

If (i) holds, then since $F_{\alpha}=\bigcap_{i=1}^{k}\left(X \backslash D_{i}\right)=X \backslash \cup_{i=1}^{k} D_{i},|\mu|\left(\cup_{i=1}^{k} D_{i}\right) \geqslant \delta$ holds. Hence, $|\mu|\left(D_{i}\right) \geqslant \delta / k$ for some $1 \leqslant i \leqslant k$. $\psi_{i}$ is one-to-one on $D_{i}$, and $\psi_{i}\left(D_{i}\right) \cap$ $\psi_{i}\left(X \backslash D_{i}\right)=\varnothing$, and hence $\left\|\mu \circ \psi_{i}\right\| \geqslant \delta / k$.

If (ii) holds, then $\left\|\mu / F_{\alpha}\right\| \geqslant 1-\delta$, and $\left\{\varphi_{j}\right\}_{j=1}^{2(n-k)+1}$ is a $1 / r$ measure separating family on $F_{\alpha}($ where $r=2(n-k)+1)$. Hence $\left\|\mu / F_{\alpha} \circ \varphi_{j}\right\| \geqslant(1-\delta) \cdot 1 / r$ for some $1 \leqslant j \leqslant r$. Also, $|\mu|\left(X \backslash F_{\alpha}\right)<\delta$ and thus $\left\|\mu / x_{X \backslash F_{\alpha}} \circ \varphi_{j}\right\|<\delta$ too. It follows that

$$
\left\|\mu \circ \varphi_{j}\right\| \geqslant\left\|\mu / F_{\alpha} \circ \varphi_{j}\right\|-\left\|\mu /{ }_{X \backslash F_{\alpha}} \circ \varphi_{j}\right\|>(1-\delta) \cdot 1 / r-\delta=\frac{1}{r}-\frac{r+1}{r} \delta .
$$

(Note that unlike case (i), in case (ii) we had to consider also the part of $\mu$ supported in $X \backslash F_{\alpha}$, since $\varphi_{j}\left(F_{\alpha}\right)$ and $\varphi_{j}\left(X \backslash F_{\alpha}\right)$ are not necessarily disjoint.) Thus in both cases $\|\mu \circ \tau\| \geqslant \min \{\delta / k, 1 / r-(r+1) \delta / r\}$ for some $\tau \in\left\{\psi_{i}\right\}_{i=1}^{k} \cup\left\{\varphi_{j}\right\}_{j=1}^{2(n-k)+1}$. Hence if we take $\lambda_{n, k}=\sup _{0 \leqslant \delta \leqslant 1} \min \{\delta / k, 1 / r-(r+1) \delta / r\}$ then $\left\{\psi_{i}\right\}_{i=1}^{k} \cup$ $\left\{\varphi_{j}\right\}_{j=1}^{2(n-k)+1}$ is a $\lambda_{n, k}$ measure separating family. The sup is clearly attained at $\delta$ which satisfies the equation $\delta / k=1 / r-(r+1) \delta / r$, i.e. $\delta=k /(r+k+r k)$ and $\lambda_{n, k}=1 /(r+k+r k)=1 /(2 n+1+2 k(n-k))$. Finally, from 2.13 it follows that if $X$ is connected, locally-connected and unicoherent then $\psi_{i}(X)$ is a dendrite for $1 \leqslant i \leqslant n$.

4. Nonexistence theorems. In this section we shall show that in many cases Theorem 3 is the best possible. The precise statement is the following. 
THEOREM 4. Let $X$ be an $n$-dimensional compact metric space $(n \geqslant 2)$, and let $\left\{\psi_{i}\right\}_{i=1}^{k} \cup\left\{\varphi_{j}\right\}_{j=1}^{l}$ be a uniformly separating family on $X$ with $\left\{\varphi_{j}\right\}_{j=1}^{l} \subset C(X)$ and $\operatorname{dim} \psi_{i}(X)=1,1 \leqslant i \leqslant k, 0 \leqslant k \leqslant n$. Then $l \geqslant 2(n-k)+1$.

We shall prove this theorem in three different cases.

Case (i). $2 \leqslant n-k \leqslant 6$.

Case (ii). $n-k=1$.

Case (iii). $n-k=0$.

It turns out that the proof of each of these cases requires different methods. Moreover, in Case (iii) we shall need some additional assumptions on the space $X$. Note that the restriction $n-k \leqslant 6$ follows from the restriction $n \leqslant 6$ in Theorem 2 . An extension of Theorem 2 for all $n \geqslant 2$ would imply the validity of Theorem 4 without the restriction $n-k \leqslant 6$. We shall not consider this problem in this article.

The following theorem implies Cases (i) and (ii) of Theorem 4.

THEOREM 5. Let $X$ be an $n$-dimensional compact metric space $(n \geqslant 2)$, and let $\left\{\varphi_{j}\right\}_{j=1}^{l} \cup\{\psi\}$ be a uniformly separating family on $X$, with $\left\{\varphi_{j}\right\}_{j=1}^{l} \subset C(X)$ and $\operatorname{dim} \psi(X) \leqslant k, 1 \leqslant n-k \leqslant 6$. Then $l \geqslant 2(n-k)+1$.

Theorem 5 implies Cases (i) and (ii) of Theorem 4, since if $\left\{\varphi_{j}\right\}_{j=1}^{l}$ and $\left\{\psi_{i}\right\}_{i=1}^{k}$ are as in Theorem 4, then, by Corollary $2.2\left\{\varphi_{j}\right\}_{j=1}^{l} \cup\{\psi\}$ are as in Theorem 5, where $\psi$ : $X \rightarrow \prod_{i=1}^{k} \psi_{i}(X)$ is defined by $\psi(x)=\left(\psi_{1}(x), \psi_{2}(x), \ldots, \psi_{k}(x)\right) .\left(\operatorname{dim} \prod_{i=1}^{k} \psi_{i}(X)=k\right.$ since $\operatorname{dim} \psi_{i}(X)=1$ for $1 \leqslant i \leqslant k$.)

Proof of Theorem 5 (CASE (i)). Let $\left\{\varphi_{j}\right\}_{j=1}^{l}$ and $\psi$ be as in Theorem 5 with $2 \leqslant n-k \leqslant 6$. Then by Theorem 2.4 there exists some $y \in \psi(X)$ with $\operatorname{dim} \psi^{-1}(y)$ $\geqslant n-k$. Obviously $\left\{\varphi_{j}\right\}_{j=1}^{l}$ is a uniformly separating family on $\psi^{-1}(y)$. By Theorem $2, l \geqslant 2(n-k)+1$.

Proof of Theorem 5 (CASE (ii)). We have to show that if $\varphi_{1} \varphi_{2}$ and $\psi$ are mappings on $X$ with $\varphi_{i} \in C(X), i=1,2$, and $\operatorname{dim} \psi(X) \leqslant n-1$, then $\left\{\varphi_{1}, \varphi_{2}, \psi\right\}$ is not a uniformly separating family. Assume that $\left\{\varphi_{1}, \varphi_{2}, \psi\right\}$ is a uniformly separating family on $X$. Without loss of generality we may assume that $X$ is an $n$-dimensional Cantor manifold, and in particular that every open subset of $X$ has dimension $n$. Then none of the families $\left\{\varphi_{1}, \psi\right\}$ and $\left\{\varphi_{2}, \psi\right\}$ is uniformly separating on any set $X^{\prime} \subset X$ with nonempty interior in $X$. This follows from Theorem 6 (which will be proved independently later) since $R$ is countably 1 -dimensional. It follows that the mappings $\left(\varphi_{i}, \psi\right): X: \rightarrow R \times \psi(X)$ are 0-dimensional, $i=1$, 2. Indeed, let $t_{0} \in R$ and $y_{0} \in \psi(X)$, and assume e.g. that $\operatorname{dim}\left(\varphi_{1}, \psi\right)^{-1}\left(t_{0}, y_{0}\right) \geqslant 1$. Then $\varphi_{2}$ is a homeomorphism on $\varphi_{1}^{-1}\left(t_{0}\right) \cap \psi^{-1}\left(y_{0}\right)=L$. Thus $\varphi_{2}(L)$ contains an open interval $J \subset R$, and $\varphi_{2}^{-1}(J) \backslash L$ is an open subset of $X$. Let $X^{\prime} \subset \varphi_{2}^{-1}(J) \backslash L$ be compact with nonempty interior. We shall see that $\left\{\varphi_{1}, \psi\right\}$ is a uniformly separating family on $X^{\prime}$ which is a contradiction. Let $f \in l_{\infty}\left(X^{\prime}\right)$. Let $\hat{f} \in l_{\infty}(X)$ be an extension of $f$ with $\hat{f} / L=0$.

$\hat{f}$ admits a representation.

$\hat{f}(x)=g_{1}\left(\varphi_{1}(x)\right)+g_{2}\left(\varphi_{2}(x)\right)+g_{3}(\psi(x))$ with $g_{i} \in l_{\infty}(R), i=1,2$, and $g_{3} \in$ $l_{\infty}(\psi(X))$. We may assume without loss of generality that $g_{1}\left(t_{0}\right)=g_{3}\left(y_{0}\right)=0$. Let 
$x \in L$. Then $0=\hat{f}(x)=g_{1}\left(\varphi_{1}(x)\right)+g_{2}\left(\varphi_{2}(x)\right)+g_{3}(\psi(x))$. But as $x \in L, \varphi_{1}(x)=$ $t_{0}$ and $\psi(x)=y_{0}$, i.e.

$$
0=\hat{f}(x)=g_{1}\left(t_{0}\right)+g_{2}\left(\varphi_{2}(x)\right)+g_{3}\left(y_{0}\right)=g_{2}\left(\varphi_{2}(x)\right) .
$$

Hence $g_{2}$ vanishes on $\varphi_{2}(L)$. But $\varphi_{2}\left(X^{\prime}\right) \in J \subset \varphi_{2}(L)$, and thus $\varphi_{2}$ vanishes on $\varphi_{2}\left(X^{\prime}\right)$ too, i.e. for $x \in X^{\prime}$ we have $f(x)=\hat{f}(x)=g_{1}\left(\varphi_{1}(x)\right)+g_{3}(\psi(x))$, which means that $\left\{\varphi_{1}, \psi\right\}$ is a uniformly separating family on $X^{\prime}$.

From the fact that $\left(\varphi_{1}, \psi\right)$ and $\left(\varphi_{2}, \psi\right)$ are 0-dimensional, it follows that both $\varphi_{1}$ and $\varphi_{2}$ are 0 -dimensional on $\psi^{-1}(y)$ for each $y \in \psi(X) . \psi$ itself is a 1-dimensional mapping. (dim $\psi=0$ would imply $\operatorname{dim} \psi(X) \geqslant \operatorname{dim} X=n$, while $\operatorname{dim} \psi^{-1}(y) \geqslant 2$ for some $y \in \psi(X)$ is impossible by Theorem 2 since $\left\{\varphi_{1}, \varphi_{2}\right\}$ is uniformly separating on $\psi^{-1}(y)$.) Hence by Theorem 2.5 the set $Y=\left\{y \in \psi(X): \operatorname{dim} \psi^{-1}(y) \geqslant 1\right\}$ has dimension $\geqslant n-1$ and in particular $|Y|>\boldsymbol{\aleph}_{0}$.

We shall construct now two disjoint sequences $\left\{x_{j}^{\prime}\right\}_{j=1}^{m}$ and $\left\{x_{j}^{\prime \prime}\right\}_{j=1}^{m}$ of arbitrary length $m$ in $X$, so that for each $j, \psi\left(x_{j}^{\prime}\right)=\psi\left(x_{j}^{\prime \prime}\right)$,

$$
\varphi_{1}\left(x_{2 j-1}^{\prime}\right)=\varphi_{1}\left(x_{2 j}^{\prime}\right), \quad \varphi_{1}\left(x_{2 j-1}^{\prime \prime}\right)=\varphi_{1}\left(x_{2 j}^{\prime \prime}\right), \quad j \geqslant 1,
$$

and

$$
\varphi_{2}\left(x_{2 j}^{\prime}\right)=\varphi_{2}\left(x_{2 j+1}^{\prime}\right), \quad \varphi_{2}\left(x_{2 j}^{\prime \prime}\right)=\varphi_{2}\left(x_{2 j+1}^{\prime \prime}\right), \quad j \geqslant 1 .
$$

Once we do this, we can define $\mu \in l_{1}(X)$ by $\mu=\sum_{j=1}^{m}(-1)^{j} \delta_{x_{j}^{\prime}}-\sum_{j=1}^{m}(-1)^{j} \delta_{x_{j}^{\prime \prime}}$ (where $\delta_{x}$ is the Dirac measure with mass 1 at $x$ ). Then $\|\mu\|=2 m, \mu \circ \psi=0$, and $\left\|\mu \circ \varphi_{i}\right\| \leqslant 4$ for $i=1,2$, i.e. $\|\mu \circ \tau\| \leqslant 2 / m\|\mu\|$ for all $\tau \in\left\{\varphi_{1}, \varphi_{2}, \psi\right\}$ which shows that $\left\{\varphi_{1}, \varphi_{2}, \psi\right\}$ is not a uniformly separating family.

Let $B$ be a countable basis for the topology of $R$. For $J \in B$ let $Y_{J}=\{y \in Y$ : there exists a compact connected set $W_{y} \subset \psi^{-1}(y)$ so that $\left.J \subset \varphi_{1}\left(W_{y}\right)\right\}$. Then $Y=\cup_{J \in B} Y_{J}$. Indeed, let $y \in Y$; then since $\operatorname{dim} \psi^{-1}(y) \geqslant 1, \psi^{-1}(y)$ contains some nontrivial connected compact set $W_{y}$, and since $\operatorname{dim} \varphi_{1}=0$ on $\psi^{-1}(y), \operatorname{dim} \varphi_{1}\left(W_{y}\right)$ $=1$ and hence $\varphi_{1}\left(W_{y}\right)$ as a 1-dimensional subset of $R$ contains some $J \in B$, i.e. $y \in Y_{J}$. It follows that for some $J \in B,\left|Y_{J}\right|>\boldsymbol{\aleph}_{0}$. Note also that $J \times Y_{J} \subset$ $\left(\varphi_{1}, \psi\right)(X)$. (If $t \in J$ and $y \in Y_{J}$, then $t \in J \subset \varphi_{1}\left(\psi^{-1}(y)\right.$ ); hence for some $x \in$ $\psi^{-1}(y), \varphi_{1}(x)=t$ and thus $\left(\varphi_{1}, \psi\right)(x)=(t, y)$.) Let $J^{\prime}$ and $J^{\prime \prime}$ be two open intervals in $J$ with $\bar{J}^{\prime} \cap \bar{J}^{\prime \prime}=\varnothing$, and set $X^{\prime}=\varphi_{1}^{-1}\left(\bar{J}^{\prime}\right), X^{\prime \prime}=\varphi_{1}^{-1}\left(\overline{J^{\prime \prime}}\right)$. Then $X^{\prime} \cap X^{\prime \prime}=\varnothing$, $\operatorname{dim} X^{\prime}=\operatorname{dim} X^{\prime \prime}=n$ (since both have nonempty interior in $X$ ) and for all $y \in Y_{J}$, $\operatorname{dim}\left(X^{\prime} \cap \psi^{-1}(y)\right)=\operatorname{dim}\left(X^{\prime \prime} \cap \psi^{-1}(y)\right)=1$. Indeed, let $y \in Y_{J}$. Then for some compact connected set $W_{y} \subset \psi^{-1}(y), \varphi_{1}\left(W_{y}\right) \supset J \supset J^{\prime}$ and hence $W_{y} \cap \varphi_{1}^{-1}\left(J^{\prime}\right) \subset X^{\prime}$ is an open set relative to $W_{y}$; and since an open subset of a compact connected set is positive dimensional, $\operatorname{dim}\left(X^{\prime} \cap \psi^{-1}(y)\right) \geqslant 1$. The same argument applies to $X^{\prime \prime}$.

Set $X_{m}^{\prime}=X^{\prime}$ and $Y_{m}^{\prime}=Y_{J}$. We shall construct a decreasing sequence of $n$ dimensional compact sets $X_{m}^{\prime} \supset X_{m-1}^{\prime} \supset \cdots \supset X_{1}^{\prime}$ and a decreasing sequence $Y_{m}^{\prime} \supset Y_{m-1}^{\prime} \supset \cdots \supset Y_{1}^{\prime}$ of uncountable sets as follows: Applying the argument which has been used to show that $\left|Y_{J}\right| \geqslant \boldsymbol{N}_{0}$ for some $J \in B$, and the fact that $\operatorname{dim}\left(X_{m}^{\prime} \cap \psi^{-1}(y)\right) \geqslant 1$ for all $y \in Y_{m}^{\prime}$, we can show that for some open interval $I \subset \varphi_{2}\left(X_{m}^{\prime}\right) \subset R$ the set $Y_{m-1}^{\prime}=\left\{y \in Y_{m}^{\prime}\right.$ : there exists a compact connected set 
$W_{y} \subset \psi^{-1}(y) \cap X_{m}^{\prime}$ so that $\left.I \subset \varphi_{2}\left(W_{y}\right)\right\}$ is uncountable. Then $Y_{m-1}^{\prime} \subset Y_{m}^{\prime},\left|Y_{m-1}^{\prime}\right|>$ $\aleph_{0}$ and $I \times Y_{m-1}^{\prime} \subset\left(\varphi_{2}, \psi\right)\left(X_{m}^{\prime}\right)$. Set $X_{m-1}^{\prime}=\varphi_{2}^{-1}(\bar{I}) \cap X_{m}^{\prime}$. Then $\operatorname{dim} X_{m-1}^{\prime}=n$, and as before, for all $y \in Y_{m}^{\prime}, \operatorname{dim}\left(X_{m-1}^{\prime} \cap \psi^{-1}(y)\right) \geqslant 1$. Next, operate on $X_{m-1}^{\prime}$ in the very same way with $\varphi_{1}$ replacing $\varphi_{2}$, to obtain $Y_{m-2}^{\prime}$ and $X_{m-2}^{\prime}$. Then operate on $X_{m-2}^{\prime}$ with $\varphi_{2}$ again to obtain $Y_{m-3}^{\prime}$ and $X_{m-3}^{\prime}$ and continue by an obvious induction till we end up with $Y_{1}^{\prime}$ and $X_{1}^{\prime}$.

Let us see now that for any $m$ points $y_{1}, y_{2}, \ldots, y_{m}$ in $Y_{1}^{\prime}$ with $m$ even, there exist $m$ points $\left\{x_{j}^{\prime}\right\}_{j=1}^{m}$ in $X_{m}^{\prime}$ so that $\psi\left(x_{j}^{\prime}\right)=y_{j}, \varphi_{1}\left(x_{2 j-1}^{\prime}\right)=\varphi_{1}\left(x_{2 j}^{\prime}\right)$ and $\varphi_{2}\left(x_{2 j}^{\prime}\right)=$ $\varphi_{2}\left(x_{2 j+1}^{\prime}\right)$. So, let $\left\{y_{j}\right\}_{j=1}^{m} \subset Y_{1}^{\prime}$. By the construction, there exists some interval $I \subset \varphi_{1}(X)$, so that $X_{1}^{\prime}=\varphi_{1}^{-1}(\bar{I})$ and $I \times Y_{1}^{\prime} \subset\left(\varphi_{1}, \psi\right)\left(X_{2}^{\prime}\right)$. Let $x_{1}^{\prime} \in X_{1}^{\prime}$ be such that $\psi\left(x_{1}^{\prime}\right)=y_{1}$. (Such a point clearly exists. Actually $\operatorname{dim}\left(X_{1}^{\prime} \cap \psi^{-1}\left(y_{1}\right)\right) \geqslant 1$.) Set $t_{1}=$ $\varphi_{1}\left(x_{1}^{\prime}\right)$. Then $t_{1} \in I$, and $y_{1}, y_{2}$ are in $Y_{1}^{\prime}$. Hence $\left(t_{1}, y_{1}\right)$ and $\left(t_{1}, y_{2}\right)$ are both in $I \times Y_{1}^{\prime} \subset\left(\varphi_{1}, \psi\right)\left(X_{2}^{\prime}\right)$. It follows that there exists some $x_{2}^{\prime} \in X_{2}^{\prime}$ with $\left(\varphi_{1}, \psi\right)\left(x_{2}^{\prime}\right)=$ $\left(t_{1}, y_{2}\right)$, i.e. $\psi\left(x_{2}^{\prime}\right)=y_{2}$ and $\varphi_{1}\left(x_{2}^{\prime}\right)=\varphi_{1}\left(x_{1}^{\prime}\right)=t_{1}$. Let $s_{2}=\varphi_{2}\left(x_{2}^{\prime}\right)$. By the construction $X_{2}^{\prime}=\varphi_{2}^{-1}(\bar{J})$ where $J \subset R$ is some interval so that $J \times Y_{2}^{\prime} \subset\left(\varphi_{2}, \psi\right)\left(X_{3}^{\prime}\right)$. Since $Y_{2}^{\prime} \supset Y_{1}^{\prime}$ we can apply the same argument to find some $x_{3}^{\prime} \in X_{3}^{\prime}$ with $\psi\left(x_{3}^{\prime}\right)=y_{3}$ and $\varphi_{2}\left(x_{3}^{\prime}\right)=\varphi_{2}\left(x_{2}^{\prime}\right)=s_{2}$. Inductively we continue and construct $x_{4}^{\prime}, \ldots, x_{m}^{\prime}$ which satisfy our conditions. Let us return now to $X^{\prime \prime}$. Since $Y_{1}^{\prime} \subset Y_{J}=Y_{m}^{\prime}, \operatorname{dim}\left(X^{\prime \prime} \cap \psi^{-1}(y)\right) \geqslant 1$ for all $y \in Y_{1}^{\prime}$. Hence we can repeat the same process with $X^{\prime \prime}$ replacing $X^{\prime}$ and $Y_{1}^{\prime}$ replacing $Y_{J}$. In this way we obtain sets $Y_{m}^{\prime \prime} \supset Y_{m-1}^{\prime \prime} \supset \cdots \supset Y_{1}^{\prime \prime}$ with $\left|Y_{j}^{\prime \prime}\right|>\aleph_{0}$, $\left(Y_{m}^{\prime \prime}=Y_{1}^{\prime}\right)$ and $X^{\prime \prime}=X_{m}^{\prime \prime} \supset X_{m-1}^{\prime \prime} \supset \cdots \supset X_{1}^{\prime \prime}$. The same argument as above shows then that for any $m$ points $\left\{y_{j}\right\}_{j=1}^{m} \subset Y_{1}^{\prime \prime}\left(m\right.$ even) there exists $m$ points $\left\{x_{j}^{\prime \prime}\right\}_{j=1}^{m} \subset$ $X_{m}^{\prime \prime}=X^{\prime \prime}$ with $\psi\left(x_{j}^{\prime \prime}\right)=y_{j}$ as above. But since $\left\{y_{j}\right\}_{j=1}^{m} \subset Y_{1}^{\prime \prime} \subset Y_{m}^{\prime \prime}=Y_{1}^{\prime}$, we can also construct such points $\left\{x_{j}^{\prime}\right\}_{j=1}^{m}$ with $\psi\left(x_{j}^{\prime}\right)=y_{j}$ in $X_{m}^{\prime}=X^{\prime}$. Then $\left\{x_{j}^{\prime}\right\}_{j=1}^{m} \cap\left\{x_{j}^{\prime \prime}\right\}_{j=1}^{m}$ $=\varnothing\left(\right.$ since $\left.X^{\prime} \cap X^{\prime \prime}=\varnothing\right)$ and we are done. This proves Case (ii) of Theorem 5 and also of Theorem 4.

The following theorem implies Case (iii) of Theorem 4, under some additional assumptions on the space $X$.

THEOREM 6. Let $X$ be an $n$-dimensional compact metric space $(n \geqslant 2)$. Let $\Psi_{i}$ : $X \rightarrow Y_{i}$ be mappings with $\operatorname{dim} Y_{i}=n_{i}<n, i=1,2$, and $n_{1}+n_{2} \leqslant n$. If $Y_{i}$ is countably $n_{i}$-dimensional (see §2) for $i=1$ or $i=2$ then $\left\{\psi_{1}, \psi_{2}\right\}$ is not a uniformly separating family.

Proof. Assume that $\left\{\psi_{1}, \psi_{2}\right\}$ is a uniformly separating family. Then $\left(\psi_{1}, \psi_{2}\right)$ : $X \rightarrow Y_{1} \times Y_{2}$ is an embedding, and hence $W=\left(\psi_{1}, \psi_{2}\right)$ is an $n$-dimensional compact subset of $Y_{1} \times Y_{2}$. By Theorem $2.14 W$ contains a product $Y_{1}^{\prime} \times Y_{2}^{\prime} \subset W$ with $\operatorname{dim} Y_{i}^{\prime}=n_{i}, i=1,2$. Let $\alpha_{1}, \alpha_{2}$ be points in $Y_{1}^{\prime}$ and $\beta_{1}, \beta_{2}$ points in $Y_{2}^{\prime}$. Then $W$ contains the points $\left(\alpha_{1}, \beta_{1}\right),\left(\alpha_{1}, \beta_{2}\right),\left(\alpha_{2}, \beta_{1}\right)$ and $\left(\alpha_{2}, \beta_{2}\right)$. Let $\left\{x_{j}\right\}_{j=1}^{4} \subset X$ be points so that

$$
\begin{gathered}
\left(\psi_{1}, \psi_{2}\right)\left(x_{1}\right)=\left(\alpha_{1}, \beta_{1}\right), \quad\left(\psi_{1}, \psi_{2}\right)\left(x_{2}\right)=\left(\alpha_{2}, \beta_{2}\right), \\
\left(\psi_{1}, \psi_{2}\right)\left(x_{3}\right)=\left(\alpha_{1}, \beta_{2}\right) \quad \text { and } \quad\left(\psi_{1}, \psi_{2}\right)\left(x_{4}\right)=\left(\alpha_{2}, \beta_{1}\right)
\end{gathered}
$$

and set $\mu=\delta_{x_{1}}+\delta_{x_{2}}-\delta_{x_{3}}-\delta_{x_{4}}$. Then $\mu \in l_{1}(X),\|\mu\|=4$ and $\mu \circ \psi_{i}=0$ for $i=1,2$, which shows that $\left\{\psi_{1}, \psi_{2}\right\}$ is not a uniformly separating family. 
Proof of Theorem 4 (CASe (iii)) Under the assumption that $X$ is CONNected, LOCALLY-CONNECTED AND UNICOHERENT. Let $X$ be as above, and let $\left\{\psi_{i}\right\}_{i=1}^{n}$ be mappings on $X$ so that $\operatorname{dim} \psi_{i}(X)=1,1 \leqslant i \leqslant n$. We wish to show that $\left\{\psi_{i}\right\}_{i=1}^{n}$ is not a uniformly separating family on $X$. If it is, then by Corollary 2.8 we may assume that the $\psi_{i}$ 's are monotone mappings. From 2.13 it follows then that $\psi_{i}(X)$ is a dendrite for $1 \leqslant i \leqslant n$, and by $2.15 \psi_{i}(X)$ is countably 1-dimensional. Set $\psi=\left(\psi_{2}, \psi_{3}, \ldots, \psi_{n}\right): X \rightarrow \prod_{i=2}^{m} \psi_{i}(X)$. Then $\operatorname{dim} \psi(X) \leqslant n-1$, and by $2.2\left\{\psi_{1}, \psi\right\}$ is a uniformly separating family, contradicting Theorem 6 .

Corollary. Let $X$ be a 2-dimensional compact, connected, locally-connected and unicoherent space, and let $\left\{\psi_{i}\right\}_{i=1}^{m}$ be a uniformly separating family on $X$, with $\operatorname{dim} \varphi_{i}(X)<\operatorname{dim} X, 1 \leqslant i \leqslant m$. Then $\sum_{i=1}^{m} E\left(\varphi_{i}(X)\right) \geqslant 5$ where $E$ is the Euclidean index defined in $\$ 1$.

Proof. Assume that for $k \varphi_{i}$ 's, $E\left(\varphi_{i}(X)\right) \geqslant 2$, and for $l \varphi_{i}$ 's, $E\left(\varphi_{i}(X)\right)=1$ (i.e. $\left.\varphi_{i} \in C(X)\right)$ where $k+l=m$. If $k=0$ then by Theorem 4, Case (i), $l \geqslant 5$ and thus $\sum_{i=1}^{m} E\left(\varphi_{i}(X)\right) \geqslant 5$. If $k=1$ then by Case (ii) of the same theorem $l \geqslant 2(2-1)+1$ $=3$ and $\sum_{i=1}^{m} E\left(\varphi_{i}(X)\right) \geqslant 2+3=5$. If finally $k=2$ then by Case (iii), $l \geqslant 1$ (here the special assumptions on $X$ are applied) and again $\sum_{i=1}^{m} E\left(\varphi_{i}(X)\right) \geqslant 2 \cdot 2+1=5$. For $k \geqslant 3$ the corollary obviously holds.

\section{REFERENCES}

1. V. I. Arnold, Representation of continuous functions of three variables, Amer. Math. Soc. Transl. (2) 28 (1963), 61-149.

2. C. F. K. Jung, Mappings on compact metric spaces, Colloq. Math. 19 (1968), 73-76.

3. J. Keesling, Closed mappings which lower dimension, Colloq. Math. 20 (1969), 237-241.

4. A. N. Kolmogorov, On the representation of continuous functions of many variables by superpositions of continuous functions of one variable and addition, Dokl. Akad. Nauk SSSR 114 (1959), 953-956.

5. K. Kuratowski, Topology. II, Academic Press, New York, 1968.

6. A. Lelek, On mappings that change dimension of spheres, Colloq. Math. 10 (1963), 45-48.

7. K. Menger, Kurventheorie, Teubner, Leipzig, 1932.

8. P. A. Ostrand, Dimension of metric spaces and Hilbert's problem 13, Bull. Amer. Math. Soc. 7 (1965), 619-622.

9. Y. Sternfeld, Uniformly separating families of functions, Israel J. Math. 29 (1978), 61-91.

10. __ Superpositions of continuous functions, J. Approx. Theory 25 (1979), 360-368.

11. Dimension of subsets of product spaces, Proc. Amer. Math. Soc. 82 (1981), 452-454.

12. Uniformly separating families of functions. II, preprint.

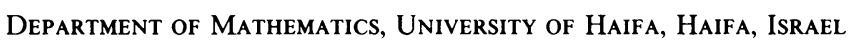

